\title{
ROLE OF IONIC PROCESSES IN DEGRADATION OF WIDE-GAP II-VI SEMICONDUCTOR MATERIALS
}

\author{
L.V. Borkovskaya, B.R. Dzhymaev, N.E. Korsunskaya, \\ I.V. Markevich, A.F. Singaevsky and M.K. Sheinkman \\ Institute of Semiconductor Physics, National Academy of Sciences of Ukraine \\ Prospect Nauki 45, Kiev, 252028, Ukraine
}

\begin{abstract}
A role of mobile defects in processes responsible for II-VI compound semiconductor characteristic instability is under consideration. These defects have been shown to be responsible for electron-enhanced reactions in these materials, in particular, shallow donor creation in CdS crystals. Accumulation of mobile defects near dislocations results in some specific effects: anisotropy of conductivity induced by electric field and distortion of edge emission spectrum shape. These effects side by side with electron-enhanced defect reactions have been found to influence considerably semiconductor device characteristics.
\end{abstract}

PACS numbers: 71.55.Jv, 78.55.Et

\section{Introduction}

In spite of high photosensitivity and intensive photoluminescence in broad spectral range II-VI compounds do not have a wide practical application up to now. The main reason of this situation is the nonstability of these material characteristics which causes a rapid degradation of II-VI light-emitting devices.

Our investigations have shown that the main factor of II-VI compound semiconductor characteristic nonstability is the presence of mobile defects. It has been proved that a change of these defect interactions with one another and with other defects as well as their replacement in crystal under different external factors result in electric, photoelectric, and photoluminescent material characteristic changes and some specific effects $[1,2]$.

For mobile defect detection a method based on their drift under electric field has been elaborated. When an electric field is applied to the sample at a suitable temperature, charged mobile defects, if they are present, will move to the electrodes, donors being collected near the cathode and acceptors being accumulated near the anode. Investigations of electric and photoelectric characteristics as well as exciton luminescence spectra of sample areas near electrodes before and 
after electric field application allow the detection of such mobile defects and their nature identification. Diffusion activation energy of the drifting defects can be obtained from the temperature dependençe of the drift rate [3]. In CdS, CdSe, CdSSe crystals a number of defects mobile in $250-350 \mathrm{~K}$ temperature range has been found. These defects have been shown to be both impurity $\left(\mathrm{Cu}_{\mathrm{i}}, \mathrm{Li}_{\mathrm{i}}\right)$ and intrinsic $\left(\mathrm{Cd}_{\mathrm{i}}, \mathrm{S}_{\mathrm{i}}\right)$ interstitials. It has been proved that there are these defects that are responsible for electron-enhanced defect reactions (EEDR) such as donor-acceptor pair dissociation and creation of point defect association.

In CdS crystals the EEDR resulting in shallow donor creation have also been found. It should be noticed that defect reactions connected with shallow donor appearance (or disappearance) attract an intent attention of investigators for a long time. Such processes are observed in $\mathrm{A}_{2} \mathrm{~B}_{6}, \mathrm{~A}_{3} \mathrm{~B}_{5}, \mathrm{Ge}$, and $\mathrm{Si}$ semiconductors and are explained as a rule as local point defect rearrangement. In this work the different processes of shallow donor creation connected with mobile defect long distance diffusion have been investigated.

\section{Results and discussion}

Nominally undoped bulk highly resistive $\left(p=10^{8} \div 10^{12} \Omega \mathrm{cm}\right)$ photosensitive CdS crystals grown by zone sublimation have been studied. Samples were cut off from the bowl and then ohmic indium contacts were melted on the cleaved surface.

An increase in equilibrium conductivity and photosensitivity as well as thermally stimulated current (TSC) spectrum changes were observed after illumination of these crystals with intrinsic light in the $350-400 \mathrm{~K}$ temperature range. After heating up to $450 \mathrm{~K}$ and subsequent cooling in dark the initial state restores.

It has been found from the TSC investigations that after the reaction carried out the density of shallow electron traps responsible for the peak at $40 \mathrm{~K}$

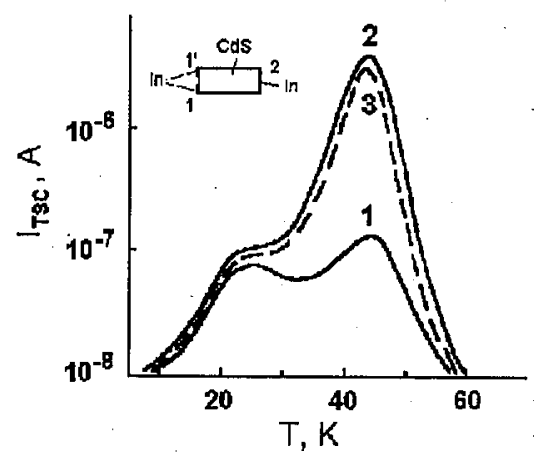

Fig. 1. Thermally stimulated conductivity spectra measured between 1 and $1^{\prime}$ electrodes: 1 - in the initial state (cooling from $450 \mathrm{~K}$ in dark), 2 - after the reaction carried out (illumination with intrinsic light in the 400-350 $\mathrm{K}$ temperature range), 3 - after mobile defect drift (cooling in dark from $450 \mathrm{~K}$ under the electric field $E=5 \times 10^{2} \mathrm{~V} / \mathrm{cm}$ applied between electrodes $\left(1,1^{\prime}\right)$-2, electrodes $1,1^{\prime}$ being cathode). Overhead on the left: the sample scheme. 
increases (Fig. 1, curves 1,2). This center ionization energy obtained from TSC curves is equal to $0.03 \mathrm{eV}$. It has been found that these centers drift from anode to cathode and accumulate near cathode under electric field $E=10^{2} \div 10^{3} \mathrm{~V} / \mathrm{cm}$ (Fig. 1, curve 3). Simultaneously the increase in intensity of $I_{2}$ line $\lambda=0.48695 \mathrm{~nm}$ which is known to be due to exciton bound on $\mathrm{Cd}_{\mathrm{i}}$ [3] has been observed in exciton luminescence spectrum in the near cathode region. Therefore, the centers which are created in the result of reaction are hydrogen-like $\mathrm{Cd}_{\mathrm{i}}$ donors. Their diffusion activation energy determined from the drift rate temperature dependence [3] is equal to $0.4 \mathrm{eV}$ both in dark and under illumination (Fig. 2, curve 3).

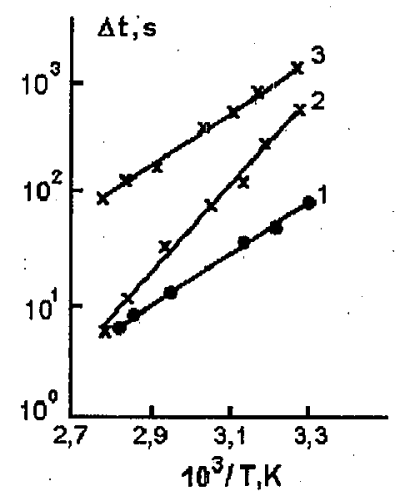

Fig. 2. Temperature dependences of reciprocal rate of: 1 - reaction process, 2 - initial state restoration, $3-\mathrm{Cd}_{\mathrm{i}}$ drift.

The investigation of reaction spectrum (spectral distribution of light that gives rise to the reaction) has shown that it coincides with the photocurrent spectrum, the reaction rate being directly proportional to the photoelectron density.

The activation energies of reaction $E_{\mathrm{r}}$ and the initial state restoration $E_{\mathrm{i}}$ determined from the rate temperature dependence of each process [4] have been found to be equal to $0.75 \pm 0.05$ and $0.4 \pm 0.05 \mathrm{eV}$, respectively. Since the latter value coincides with $\mathrm{Cd}_{\mathrm{i}}$ diffusion activation energy, one can suppose that the process under consideration is due to $\mathrm{Cd}_{\mathrm{i}}$ bulk diffusion.

The detailed investigation of photocurrent, photoluminescence, and TSC spectra in the 80-450 K temperature range has shown that shallow donor creation is accompanied by neither appearance nor disappearance of any other traps or recombination centers as it takes place in other EEDR observed in these crystals and caused by rearrangement of point defect associates. At the same time CdS crystals are known to include cadmium clusters of various sizes [5]. Besides, it has been found that $\mathrm{Cd}_{\mathrm{i}}$ atoms are accumulated near dislocations creating highly conductive regions $[2,6]$. The latter reveal themselves in shallow donor EPR spectra as well as dependence of conductivity on applied voltage frequency (amplitude-frequency characteristic, AFC) [2]. Therefore, both clusters and near dislocation regions can be sources of $\mathrm{Cd}_{\mathrm{i}}$ atoms. In the latter case conductivity of near dislocation regions should be expected to decrease or, at least, to remain unchanged. However, 
the measurement of $\mathrm{AFC}$ has shown that conductivity of near dislocation regions increases after the reaction carried out. Thus, it may be thought that $\mathrm{Cd}_{\mathrm{i}}$ atoms originate from cadmium clusters which are present both in crystal bulk and in near dislocation regions. Cluster recharge after photoelectron capture gives rise to its whole or partial dissociation accompanied by the release of $\mathrm{Cd}_{\mathrm{i}}$ atoms. The latter diffuse in the crystal bulk, which leads to the increase in shallow donor density. The activation energy of this process is, obviously, determined by $\mathrm{Cd}_{\mathrm{i}}$ bonding energy in the cluster after its recharge. In dark reverse $\mathrm{Cd}_{\mathbf{i}}$ atom diffusion towards residual nucleus and initial defect restoration take place.

The presence of clusters can reveal itself in crystal optical characteristics, particularly, light scattering ones. It is well known that defect clusters can transform due to thermal treatment [7], so we have investigated the influence of annealing on light scattering spectrum as well as on reaction intensity. The sample has been annealed at $500 \mathrm{~K}$ during 10 minutes in situ and then quenched to room temperature, which promotes to cluster destruction [7]. It has been found that after such annealing light scattering in the $500-700 \mathrm{~nm}$ wavelength range decreases (Fig. 3a). At the same time the value of TSC peak at $40 \mathrm{~K}$ practically does not change in the initial state (Fig. $3 \mathrm{~b}$, curves 1,2 ) and considerably increases after the reaction carried out (Fig. 3b, curves $1^{\prime}, 2^{\prime}$ ).
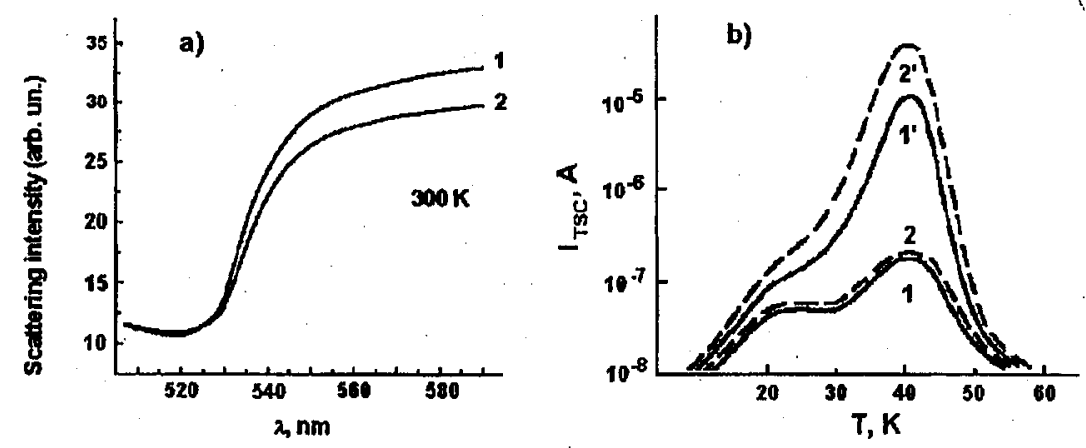

Fig. 3. (a) Light scattering spectra at $300 \mathrm{~K}$ before (1) and after (2) annealing. (b) Thermally stimulated conductivity spectra of the same sample before $\left(1,1^{\prime}\right)$ and after $\left(2,2^{\prime}\right)$ annealing in initial state $(1,2)$ and after the reaction carried out $\left(1^{\prime}, 2^{\prime}\right)$.

Since only rather large clusters make contribution to light scattering, it may be thought that this cluster density decreases due to annealing. This process, however, does not result in $\mathrm{Cd}_{\mathrm{i}}$ atom density increase. Hence, one can suppose that under annealing large clusters fall to smaller ones which do not reveal themselves in light scattering in the investigated wavelength range, and which can dissociate after photoelectron capture. A small cluster density increase results in the reaction intensity rise after annealing.

It should be noted that clusters of intrinsic defects are present in the most semiconductor materials both in the single crystals and in MBE layers (see, for instance, $[8,9])$. Therefore one may think that the above-mentioned process can 
take place in other semiconductors too. Besides, cluster formation and transformation change potential relief which can result in persistent photoconductivity and other conductivity anomalies which are often considered to indicate DX-like center presence.

Our investigations have shown that side by side with EEDR other effects can be caused by the mobile defect presence. In CdS crystals we have observed some specific phenomena due to the mobile defect replacement. Two of these phenomena which call forth a considerable change of semiconductor characteristics are: (i) induced by external electric field anisotropy of conductivity; this effect has been shown to be caused by the formation of conductive channels due to the dislocation decoration with mobile shallow donors [10]; (ii) edge emission spectrum shape distortion due to the absorption edge prolongation as a result of shallow donor and acceptor accumulation near dislocations. This effect is responsible for degradation of working element characteristics of CdS electron-beam-pumped lasers, namely, to the shift of the emitted band and its intensity decrease during the operation.

\section{References}

[1] M.K. Sheinkman, N.E. Korsunskaya, I.V. Markevich, T.V. Torchinskaya, J. Phys. Chem. Solids 43, 475 (1982).

[2] M.K. Sheinkman, N.E. Korsunskaya, B.R. Dzhumaev, I.V. Markevich, A.F. Singaevsky, L.V. Borkovskaya, in: 23th Int. Conf. on Physics of Semiconductors, Berlin 1996, Eds. M. Scheffler, R. Zimmermann, World Scientific, Singapore 1996, p. 2985.

[3]' N.E. Korsunskaya, I.V. Markevich, I.Yu. Shabliy, M.K. Sheinkman, Fiz. Tekh. Poluprovodn. 15, 279 (1981).

[4] L.V. Borkovskaya, B.R. Dzhymaev, B. Embergenov, N.E. Korsunskaya, I.V. Markevich, Ukr. Fiz. Jurn., in print.

[5] L.I. Khandros, G.S. Pekar, M.K. Sheinkman, E.L. Shtrum, Phys. Status Solidi A 33, 765 (1976); ibid. 34, 397 (1976).

[6] G. Doding, R. Labusch, Phys. Status Solidi A 68, 143 (1981).

[7] F.A. Kröger, The Chemistry of Imperfect Crystals, North-Holland, Amsterdam 1964.

[8] A.C. Warren, J.M. Woodall, J.L. Frecouf, D. Grischkowsky, D.T. Malnturff, M.L. Melloch, Appl. Phys. Lett. 57, 1331 (1990).

[9] M. Reiche, J. Reiche, W. Nitzche, Phys. Status Solidi A 107, 851 (1988).

[10] I.A. Drozdova, B. Embergenov, N.E. Korsunskaya, I.V. Markevich, A.F. Singaevski, Fiz. Tekh. Poluprovodn. 29, 277 (1995). 